\title{
Additive Sanitization: A Technique for Pattern- Preserving Anonymization for Time-Series Data
}

\author{
Revathi.S \\ PG Scholar \\ Sri Ramakrishna Engineering College \\ Tamil Nadu, India
}

\author{
Jeyalakshmi.I \\ Assistant Professor \\ Sri Ramakrishna Engineering College \\ Tamil Nadu, India
}

\begin{abstract}
A time series is a set of data normally collected at usual intervals and often contains huge amount of individual privacy. The need to protect privacy and anonymization of time-series while trying to support complex queries such as pattern range and pattern matching queries. The conventional $(\mathrm{k}, \mathrm{p})$-anonymity model cannot effectively address this problem as it may suffer serious pattern loss. In the proposed work a new technique called additive sanitization has been developed which increment the supports of item sets and their subsets in order to reduce pattern loss and prevent linkage attack.
\end{abstract}

\section{Keywords}

Time series, pattern, sanitization, privacy, anonymity

\section{INTRODUCTION}

Privacy protection in the publication of time series is a difficult one due to the complex nature of the data. Particularly consider an essential problem of anonymizing time series while trying to support the queries. However the time sensitive attribute values and their patterns can be used as strong quasi-identifiers (QI) to launch linkage attack which reidentify some of the records. The desirable solution to prevent linkage attacks is to enforce (k, p)-anonymity on the published database, so that each record has its QI attributes identical to at least $\mathrm{k}-1$ other records. Even though the conventional $(\mathrm{k}, \mathrm{p})$-anonymity can be used to resist linkage attacks, it cannot efficiently preserve the patterns, which are significant for performing queries on time series. In this paper we develop a novel sanitization strategy, named additive, the idea is to increment supports of item sets and their subsets (by virtually adding transactions in the original dataset),minimizing distortion means reducing as much as possible the increments of supports (i.e., number of transactions virtually added).

\section{RELATED WORK}

In this sector to summarize the existing works of partial information hiding; particularly it was related to time series. The existing partial information hiding approaches can be divided into two types, the perturbation-based approaches and the partition-based approaches. Perturbation-based approaches [1], [2] protect data by adding noises. However, it does not prevent linkage attack. Partition-based approaches first divide tuples of data set into disjoint groups and then release some general information of each group.

K- anonymity [3] and condensation [4] are two approaches in this group. K-anonymity is a necessary approach to privacy preserving data publishing and generalization is the most trendy approach of enforcing k-anonymity. But it has weakness when being applied on time-series data. The limitation of condensation is that it cannot preserve the correlations of attributes for individual data. Microaggregation [5] can be used to prevent linkage attacks on time series. In addition, the data published by microaggregation also suffer pattern loss in an unrestrained manner.

\section{PROPOSED SYSTEM}

\subsection{The (K, P)-anonymity model}

Table 1: A Published Data Set T_Conforming to (k,P)Anonymity

\begin{tabular}{|l|l|l|l|l|l|l|}
\hline $\begin{array}{l}\text { Group } \\
\text { ID }\end{array}$ & $\mathbf{2 0 0 5}$ & $\mathbf{2 0 0 6}$ & $\mathbf{2 0 0 7}$ & $\mathbf{2 0 0 8}$ & $\mathbf{2 0 0 9}$ & PR \\
\hline $\mathbf{1}$ & {$[117-$} & {$[107-$} & {$[87-$} & {$[74-$} & {$[51-$} & Aabbcc \\
& $176]$ & $181]$ & $188]$ & $197]$ & $213]$ & \\
\hline $\mathbf{1}$ & {$[117-$} & {$[107-$} & {$[87-$} & {$[74-$} & {$[51-$} & Aabbcc \\
& $176]$ & $181]$ & $188]$ & $197]$ & $213]$ & \\
\hline $\mathbf{1}$ & {$[117-$} & {$[107-$} & {$[87-$} & {$[74-$} & {$[51-$} & Ccbbaa \\
& $176]$ & $181]$ & $188]$ & $197]$ & $213]$ & \\
\hline $\mathbf{2}$ & {$[32-$} & {$[54-$} & {$[47-$} & {$[38-$} & {$[20-$} & Abbbcc \\
& $98]$ & $120]$ & $125]$ & $132]$ & $161]$ & \\
\hline $\mathbf{1}$ & {$[117-$} & {$[107-$} & {$[87-$} & {$[74-$} & {$[51-$} & Ccbbaa \\
& $176]$ & $181]$ & $188]$ & $197]$ & $213]$ & \\
\hline $\mathbf{2}$ & {$[32-$} & {$[54-$} & {$[47-$} & {$[38-$} & {$[20-$} & Abbbcc \\
& $98]$ & $120]$ & $125]$ & $132]$ & $161]$ & \\
\hline
\end{tabular}

In Table 1 the published data set to preserve the pattern information for each time series, there is an additional PR column which implements an alphabetic string representation. Here the QI attributes are generalized based on the formed kgroup $(\mathrm{k}=4)$. In each $\mathrm{k}$-group, the PR column conforms to $\mathrm{P}$ anonymity $(\mathrm{p}=2)$.

\subsection{The Utility Measures}

To generate the utility measures of $(\mathrm{k}, \mathrm{P})$-anonymity model, including the breach probability, which represents the privacy preservation ability, and the information loss, which represents the utility of published data. There are two kinds of information loss, instant value loss (VL), and the pattern loss (PL).

\subsection{Instant Value Loss Metric}

The instant value loss of $\mathrm{Q}$ is given by

$$
\mathrm{VL}(\mathrm{Q})=\sqrt{\sum\left(\mathrm{r}_{\mathrm{i}}{ }^{+}-\mathrm{r}_{\mathrm{i}}^{-}\right)^{2 / \mathrm{n}}}
$$

For database T, VL (Q) is obtained by summing up the instant value losses of all its members. 


\subsection{Pattern Loss Metric}

The pattern loss can be calculated by the distance between $\mathrm{p}(\mathrm{Q})$ and $\mathrm{p}^{*}(\mathrm{Q})$,namely

$\mathrm{PL}(\mathrm{Q})=\operatorname{distance}\left(\mathrm{p}(\mathrm{Q}), \mathrm{p}^{*}(\mathrm{Q})\right)$,

where distance(.) is a distance measure defined in the feature vector space of patterns.

\section{APPROACHES FOR ENFORCING (K, P)-ANONYMITY}

The first approach for enforcing $(\mathrm{k}, \mathrm{P})$-anonymity is to use a top-down clustering-like structure as described in the following:

1. Create first-level k-groups from the micro data set.

2. For each k-group, remove PRs from micro data based on the selected PR form. The removed PRs should minimize the pattern loss while respecting the $\mathrm{P}$ requirement within its own k-group;

3. For each k-group, make P-subgroups based on the PRs.

The second approach is a bottom-up framework to form Psubgroups from individual records first, and then construct kgroups. The bottom-up approach is described in the following: 1. Remove PRs from the micro data. The extracted PRs should minimize the pattern loss while respecting the Prequirement in the entire data set;

2. Form the second-level P-subgroups based on PRs;

\section{ADVANTAGES}

$>$ It can prevent linkage attack

$>$ This model supports customized data publishing.

\section{IMPLEMENTATION}

The (k, p)-anonymity model consists of 6 modules

\author{
1. Computing Pattern Representations \\ 2. Node Splitting \\ 3. Create-tree phase \\ 4. Recycle bad-leaves phase \\ 5. Group formation phase \\ 6. Additive sanitization
}

\subsection{Computing Pattern Representations}

When calculating pattern representations, the main aim is to attain minimal pattern loss in the published table. SAX uses a sequence of alphabets, for example, "baabccbc," to represent a time series. Given a set of alphabets a time series can be converted to an alphabet sequence as described in the following:

1. First, to specify an integer parameter level which controls granularity of the resultant SAX representation.

2. Second, to normalize the time series to have a mean of 0 and standard deviation of 1 .

\subsection{Node Splitting}

The node splitting process is followed by attempts to refine the PRs of the records in a node. For each tree node N, all its members should have identical PR .However, an increment in the level leads to changes in PRs. Therefore, the members of $\mathrm{N}$ may have different PRs at $\mathrm{N}$. level +1 . Thus, by increasing the level of $\mathrm{N}$, we can split $\mathrm{N}$ into a few partitions of records, so that each partition express records of the same PR at N. level +1 . Beginning from the root, we hold node $\mathrm{N}$ in a recursive procedure.

- If $\mathrm{N}$. size $<\mathrm{P}$, then the node is labeled as bad-leaf and the recursion terminates.

- $\quad$ Otherwise if $\mathrm{N}$. level=max-level, then the node is labeled as good leaf and the recursion terminates.

- $\quad$ Else if $\mathrm{P} \leq \mathrm{N}$. size $<2 * \mathrm{P}$, we try to maximize the level of $\mathrm{N}$ as long as all records of $\mathrm{N}$ have the identical PR.

\author{
Algorithm 1:Node splitting \\ Data:tree node N,P,max level \\ Begin \\ If N.size $<\mathrm{P}$ then \\ N.label=bad-leaf; \\ If N.level==max-level then \\ N.label =good-leaf; \\ If $\mathrm{P}<\mathrm{N}$.size $<2 * \mathrm{P}$ then \\ N.label=good-leaf; \\ Maximize N.level without node split; \\ Else \\ If $\mathrm{N}$ can be split then \\ If total size of all $\mathrm{T}$ B-nodes $>\mathrm{P}$ then \\ Generate child merge: $_{\text {m }}$ \\ child $_{\text {merge. }}$ level=N.level; \\ level of all TG-nodes is N.level +1; \\ else \\ level of all child nodes is N.level+1; \\ else \\ N.label=good-leaf; \\ End
}

\subsection{Create-tree phase}

In this phase, to produce and organize the PRs in a tree for all time series in $\mathrm{T}$ respecting the following P-requirement;

1. The root node for split is the whole data set $\mathrm{T}$;

2. The post processing step in the Naive algorithm is removed.

\subsection{Recycle bad-leaves phase}

To avoid extra suppression, we would recycle most of the bad-leaf nodes by merging them with each other. To achieve the highest PR level for each bad-leaf, we begin the recycling process from the highest PR level between all bad leaves, designated by max-bad-level.

If two bad-leaf nodes BL1 and BL2 have the same level and $\mathrm{PR}$, they can be merged into a new node, represented by $\mathrm{nm}$, which has the same level and PR. If nm contains no fewer than $\mathrm{P}$ time series, it is pointed a good-leaf, otherwise a badleaf.

\section{Algorithm 2: Recycle bad-leaves}

Data: P,leaf-list,current-level,max-bad-level

Result: P-subgroup list

Begin

Current-level=max-bad-level

While sum of all bad leaves size $\geq P$ do

If any bad leaves can merge then 
Merge them to a new node leaf-merge;

If leaf-merge.size $\geq \mathrm{P}$ then

Leaf-merge.label=good-leaf;

Else

Leaf-merge.label=bad-leaf;

Current-level--;

Suppress all time-series contained in bad leaves;

end

\subsection{Group formation phase}

The greedy k-group formation phase is described in the following steps:

Step 1: All P-subgroups in PGL containing no fewer than $\mathrm{k}$ time series are taken as k-groups and simply moved into GL.

Step 2: In the remaining P-subgroups in PGL, find the Psubgroup s1 with the minimum instant value loss, and then create a new group $\mathrm{G}=\mathrm{s} 1$.

Step 3: Find another P-subgroup $s \in$ PGL - s1, which, if merged with $\mathrm{G}$, produces the minimal value loss VL (G US).

Step 4: Repeat Step 3 until $|G| \geq k$. G is then added into GL and its respective subgroups in PGL are removed.

Step 5: Steps 2-4 are repeated until the total remaining time series in PGL are fewer than $\mathrm{k}$.

\section{Algorithm 3:Group formation}

Data:PGL,k,P

Result:Group list GL

Begin

For each P-subgroup that size $\geq 2 * \mathrm{P}$ do

Split it by top-down clustering;

If any P-subgroup that size $\geq \mathrm{k}$ then

Add it into GL and remove it from PGL;

While $|\mathrm{PGL}| \geq \mathrm{k}$ do

Find $\mathrm{s}_{1}$ and $\mathrm{G}=\mathrm{s}_{1}$

While $|\mathrm{G}|<\mathrm{k}$ do

Find $s_{\min }$ and add $s_{\min }$ into $G$;

Remove all P-subgroups in G from PGL and put G in GL;

For each remaining P-subgroup s' do

Find corresponding G' and add s' into G';

end

\subsection{Additive sanitization}

In the additive sanitization, not to actually adding transactions: this is just to emphasize that the transformed set of frequent item sets maintains database-compatibility. Besides, it will contain exactly the same item sets, but with some supports increased. In the sanitization approach the idea is to increment supports of item sets and their subsets (by virtually adding transactions in the original dataset), minimizing distortion means reducing as much as possible the increments of supports (i.e., number of transactions virtually added).

This algorithm is composed by two phases: during the first phase all maximal channels are merged as much as possible, according to Maximal Channels Merging; then the resulting set of merged channels is used in the second phase to select the item sets whose support must be increased. Therefore, according to the additive sanitization, we increase the support of the item set by k. It takes in input closed frequent item sets $\mathrm{Cl}(\mathrm{D}, \sigma)$ and maximal inference channels $\mathrm{M} \mathrm{Ch}(\mathrm{k}, \mathrm{Cl}(\mathrm{D}, \sigma))$, and returns $\mathrm{O}^{\mathrm{k}}$, which in this case is the sanitized version of $\mathrm{Cl}(\mathrm{D}, \sigma)$.

\author{
Algorithm 4: Additive Sanitization \\ Input: $\operatorname{Cl}(D, \sigma), \mu C h(k, C l(D, \sigma))$ \\ Output : $O^{k}$ \\ $S \leftarrow \emptyset ;$ \\ For all $\left(C_{I}^{J}, f_{I}^{J}\right) \in \mu C h(k, C l(D, \sigma))$ do \\ If $\exists C_{I^{\prime}}^{J^{\prime}} \in S$ s.t $C_{I}^{J}$ and $C_{I^{\prime}}^{J \prime}$ can be merged
}

Then

$S \leftarrow S \backslash\left\{C_{I^{\prime}}^{J^{\prime}}\right\} ;$

$\mathrm{S} \leftarrow S \cup\left(C_{I}^{J} \bowtie C_{I^{\prime}}^{J \prime}\right) ;$

Else

$\mathrm{S} \leftarrow S \cup\left\{C_{I}^{J}\right\} ;$

For all $\left(\mathrm{I}, \sup _{D}(\mathrm{I})\right) \in \operatorname{Cl}(D, \sigma) d o$

For all $C_{I^{\prime}}^{J^{\prime}} \in S$ s.t $\mathrm{I} \subseteq I^{\prime} \mathrm{do}$

$\sup _{D}(I) \leftarrow \sup _{D}(\mathrm{I})+\mathrm{k} ;$

$\mathrm{O}^{\mathrm{k}} \leftarrow \operatorname{Cl}(D, \sigma)$

\section{CONCLUSION}

Conclusion of this work provides a new technique called additive sanitization may greatly reduce the information loss. In the additive sanitization, not to really adding transactions: this is just to show up that the transformed set of frequent item sets maintains database-compatibility. Besides it will contain exactly the same item sets, but with some supports increased. The idea of this approach is to increment supports of item sets and their subsets (by virtually adding transactions in the original dataset), minimizing distortion means reducing as much as possible the increments of supports (i.e., number of transactions virtually added). Finally, this method concluded that by increasing the support count of item sets, it will reduce the pattern and information loss.

\section{REFERENCES}

[1] S. Papadimitriou, F. Li, G. Kollios, and P.S. Yu, "Time Series Compressibility and Privacy," Proc. 33rd Int'1 Conf. Very Large Data Bases (VLDB), pp. 459-470, 2007.

[2] L. Singh and M. Sayal, "Privacy Preserving Burst Detection of Distributed Time Series Data Using Linear 
Transforms," Proc. IEEE Symp. Computational Intelligence and Data Mining (CIDM), pp. 646-653, 2007.

[3] L. Sweeney, "k-Anonymity: Privacy Protection Using Generalization and Suppression," Int'l J. Uncertainty Fuzziness and Knowledge-Based Systems, vol. 10, no. 5, pp. 571-588, 2002.

[4] C.C. Aggarwal and P.S. Yu, "A Condensation Approach to Privacy Preserving Data Mining," Proc. Ninth Int'1 Conf. Extending Database Technology (EDBT), pp. 183199, 2004.

[5] J. Nin and V. Torra, "Towards the Evaluation of Time Series Protection Methods," Information Sciences, vol. 179, no. 11, pp. 1663-1677, 2009.

[6] R. Agrawal and R. Srikant. Privacy-preserving data mining. In Proceedings of the 2000 ACM SIGMOD on Management of Data.
[7] R. Agrawal, T. Imielinski, and A. N. Swami. Mining association rules between sets of items in large databases.In Proceedings of the 1993 ACM SIGMOD.

[8] E. Dasseni, V. S. Verykios, A. K. Elmagarmid, and E. Bertino. Hiding association rules by using confidence and support. In Proceedings of the 4th International Workshop on Information Hiding, 2001.

[9] A. Evfimievski, R. Srikant, R. Agrawal, and J. Gehrke.Privacy preserving mining of association rules In Proceedings of the eighth ACM SIGKDD international conference on Knowledge discovery and data mining, 2002.

[10] S. Rizvi and J. R. Haritsa. Maintaining data privacy in association rule mining. In Proceedings of the 28th VLDB Conference, 2002. 